\title{
Expression of MUC1 and MUC4 and Its Prognostic Significance in Non-Small Cell Lung Carcinoma
}

Ji Min Jeon - Hye Won Lee Ji Young Park - Hye Ra Jung Ilseon Hwang • Sun Young Kwon Mi Sun Choe - Yu Na Kang Sang Pyo Kim - Sang Sook Lee Won Il Choi ${ }^{1} \cdot$ Kun Young Kwon

Departments of Pathology and ${ }^{1}$ Internal Medicine, Keimyung University School of Medicine, Daegu, Korea

Received : March 17, 2010

Accepted : April 1, 2010

Corresponding Author

Kun Young Kwon, M.D.

Department of Pathology, Keimyung University

School of Medicine, 194 Dongsan-dong, Jung-gu,

Daegu 700-712, Korea

Tel: 82-53-580-3812

Fax: 82-53-580-3823

E-mail:k19156ky@dsmc.or.kr

*The present research has been conducted by the Bisa Research Grant of Keimyung University in 2005.
Background : Mucin (MUC)1 and MUC4 (MUC1, 4) are high molecular weight glycoproteins expressed in normal and malignant epithelial cells, and these expressions are related to the prognosis of some carcinomas. In non-small cell lung carcinoma (NSCLC), the relationship between MUC1, 4 expressions and their prognostic significance is not well known. We evaluated these relationships in a series of NSCLC: 1) between MUC1, 4 expression levels and histologic subtypes, and 2) between high expression of MUC1, 4 and their prognostic significance. Methods : We performed immunohistochemical staining for MUC1, 4 in paraffin-embedded tissues from 165 NSCLC cases arranged in a tissue microarray. Results : We found a significant correlation between MUC1, 4 expressions and NSCLC histologic subtypes $(p<0.05)$. High MUC1 expression was characteristic of adenocarcinoma. Low MUC1, 4 expressions were characteristic of squamous cell carcinoma. In adenocarcinoma, we found significant association between diffuse MUC1 expression and short patient survival $(p=0.005)$. In squamous cell carcinoma, diffuse MUC4 expression showed long patient survival trend ( $p=$ 0.128). Conclusions : MUC1, 4 expression levels were significantly correlated with NSCLC histologic subtypes. Diffuse MUC1 expression was significantly associated with shortened survival in NSCLC patients, especially in adenocarcinoma.

Key Words : Mucin-1; MUC4 protein, human; Carcinoma, non-small cell; Prognosis
Lung cancer is one of the most common malignancies in the world, and generally has a poor prognosis. Even after complete resection of stage I tumors, approximately $30 \%$ of patients will experience disease recurrence and eventually die from the disease. ${ }^{1}$ Therefore, it is important to evaluate the malignant potential of tumor cells for more precise prediction of prognosis in patients with early-stage lung cancer. Until recently, few studies have addressed the prognostic implication of mucin (MUC) and MUC-associated antigens in lung cancer. ${ }^{2.3}$

Epithelial MUCs are major glycoprotein components of mucus, and have an established protective role in epithelial tissues. In addition, MUCs have a proposed involvement in the renewal and differentiation of the epithelium, modulation of cell adhesion and cell signaling. ${ }^{4}$ Twenty MUC-encoding genes have been characterized. ${ }^{5,6} M U C$ gene expression is organ-, tissue-, and cell type-specific. Moreover, quantitative and qualitative structural alterations and changes in the expression levels of MUCs have been observed in neoplastic lesions. These MUC-associated chan- ges have been proposed as molecular markers of malignant transformation in several organs and tissues. Furthermore, the expression levels of MUCs were related to the prognosis of breast cancer and colorectal carcinoma. ${ }^{7.8}$

MUC1 is expressed in epithelial tissues. Its expression is upregulated in breast, pancreatic, and colorectal carcinomas. ${ }^{6,9,10}$ Previous studies have reported that, high MUC1 mRNA expression in adenocarcinoma is associated with poor prognosis. ${ }^{29,10}$

MUC4 is expressed in normal epithelial tissues including tracheobronchial mucosa, colon, stomach, cervix and lung. ${ }^{4,7}$ Abnormal expression of MUC4 has been reported in pancreatic, colon, pulmonary and gastric carcinomas. ${ }^{3.811}$ MUC4 overexpression was associated with aggressiveness and metastasis in breast cancer, extrahepatic bile duct carcinoma, and cholangiocarcinoma. ${ }^{12,13}$ In contrast, improved patient survival is associated with MUC4 expression in ovarian cancer, mucoepidermoid carcinoma of the salivary glands and squamous cell carcinoma of the lung. ${ }^{3.14}$ 
To evaluate the prognostic significance and expression characteristics of MUC1 and MUC4 proteins, we attempted to determine MUC1 and MUC4 expressions semi-quantitatively by high throughput tissue microarray in a series of 165 resected non-small cell lung carcinoma (NSCLC) specimens.

\section{MATERIALS AND METHODS}

Archival tissue specimens of 243 lung cancer patients were obtained from curative excision at the Keimyung University Dongsan Hospital. Histopathologic diagnosis was performed according to the World Health Organization classification (2004) for lung cancer. ${ }^{15}$ Cases with histopathologic diagnosis of squamous cell carcinoma, adenocarcinoma, large cell carcinoma, and adenosquamous carcinoma were included in the study. Cases with mixed non-small cell and small cell lung carcinoma, giant cell carcinoma, mucoepidermoid carcinoma, sarcomatoid carcinoma, and basaloid carcinoma were excluded. Of all 243 cases, 216 cases had sufficient tissue for microarray slide analysis. Of these 216 cases, adequate clinical information was available in 165 cases.

Retrospective chart review was performed to obtain patient clinical data, including age, gender, and survival status. The tumor stage was assigned using the International Staging System for Lung Cancer. ${ }^{16}$ The median follow up period was 111 months.

\section{Construction of high-throughput tissue microarrays}

Tissue microarrays were constructed from archival formalinfixed paraffin-embedded tissues from the selected 165 patients with NSCLCs. Light microscopic examination of hematoxylin and eosin-stained sections was utilized to identify areas rich in viable tumor cells for use in tissue microarrays. A core measuring $0.5 \mathrm{~cm}$ in diameter was taken from the donor paraffin tissue blocks of each case, and arranged in a recipient paraffin tissue array block using a manual tissue arrayer (MTA-1, Beecher Instruments Inc., Sun Prairie, WI, USA).

\section{Immunohistochemistry and interpretation criteria}

Five $\mu \mathrm{m}$ sections were obtained from the tissue microarray blocks and incubated in an oven at $60^{\circ} \mathrm{C}$ overnight. Sections were dewaxed in xylene for 10 minutes, and rehydrated through a graded series of ethanol solutions to water. Endogenous per- oxidases were blocked with $3 \% \mathrm{H}_{2} \mathrm{O}_{2}$. The sections were steamed for 25 minutes in citrate buffered saline $(\mathrm{pH}$ 6.0) prior to incubation with mouse monoclonal anti-MUC1 and anti-MUC4 (1 : 750, Zymed Laboratories Inc., San Francisco, CA, USA) antibodies. Detection was performed using the Envision Plus labeled polymer system (DakoCytomation, Carpenteria, CA, USA). Nonneoplastic lung tissues were used as positive and negative control specimens. MUC1 and MUC4 expressions were assessed in a blind fashion, regardless of the patient's identity and clinical history. The extent and intensity of stained tumor cells were assessed by a semi-quantitative scale in each sample.

The extent scores of MUC1 and MUC4 staining in tumor cells were graded as follows: extent score $0(<5 \%$ immunoreactive), extent score 1 ( $<33 \%$ immunoreactive), extent score 2 (33-66\% immunoreactive), extent score 3 (> 66\% immunoreactive). The intensity scores of MUC1 and MUC4 staining in tumor cells were graded using the following scale: score 0 (negative), score 1 (weak), score 2 (moderate), score 3 (strong) (Fig. 1). A combined score (CS) was calculated by adding the extent scores and intensity scores of staining, using the following scale: CS $0=0$, CS $1=1-2$, CS $2=3-4$, CS $3=5-6$.

\section{Statistical analysis}

Statistical analysis was performed using SPSS ver. 12.0 (SPSS Inc., Chicago, IL, USA). The chi-square test was used to evaluate the association between MUC1 or MUC4 expression and the clinicopathological parameters. A p-value less than 0.05 was considered statistically significant. We performed Kaplan-Meier analysis to investigate the potential relationship between MUC1 or MUC4 expression and patient survival.

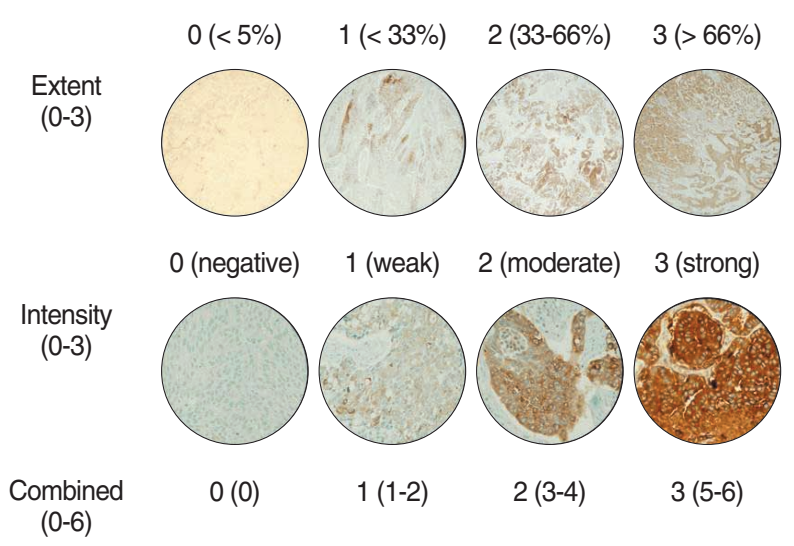

Fig. 1. The scoring criteria of the positive expression levels of mucin (MUC)1 and MUC4 immunoreactivity using tissue microarray in non-small cell lung carcinoma. 


\section{RESULTS}

\section{Clinicopathological characteristics}

The patient population consisted of 129 men (78\%) and 36 women (22\%). The mean patient age at the time of diagnosis was 60 years (range, 29 to 77 years). The most common histopathologic type was squamous cell carcinoma (59\%) followed by adenocarcinoma $(33 \%)$, large cell carcinoma (6\%), and adenosquamous carcinoma (2\%). The cases were classified into the following pathological stages: stage I (77, 47\%), II (48, 29\%), III (40, 24\%), and IV (0).

The mean survival for each histologic type were as follows: squamous cell carcinoma ( 54 months), adenocarcinoma (38 months), large cell carcinoma (21 months), and adenosquamous carcinoma (22 months).

\section{Immunohistochemical expression of MUC1 and MUC4 proteins in NSCLC}

In general, MUC1 expression was observed more frequently and at a higher intensity than MUC4 expression throughout the series. Among NSCLC, adenocarcinoma showed high expression for MUC1 (98\%) and low expression for MUC4 (29\%) on CS 2-3 (Tables 1, 2).

Squamous cell carcinoma showed diffuse MUC1 expression in the tumor cells (Fig. 2B), but focal MUC4 expression in the central portion of tumor cell nests (Fig. 2C). Adenocarcinoma cases showed diffuse positive MUC1 staining in the luminal portion and cytoplasm of tumor cells, whereas MUC4 staining was observed mainly in the apical and luminal portions of the tumor glands (Fig. 2E, F). Large cell carcinoma demonstrated diffuse positive expression of MUC1, but no expression of MUC4 (Fig. 2H, I). Adenosquamous carcinoma showed positive MUC1 expression in the tumor cells of the adenocarcinoma and squamous cell carcinoma. However, MUC4 expression was negative in the tumor cells (Fig. 2K, L). In the non-tumorous portions, MUC1 showed positive expression in the alveolar and bronchial epithelial cells, and in the submucosal glands, but was negative in the vascular endothelial cells (Fig. 2N). In contrast, MUC4 showed positive expression in the alveolar and bronchial epithelial cells, and in the vascular endothelial cells, but was negative in the submucosal glands (Fig. 2O).

Table 1. Comparison of MUC1 expression between each combined score and groupings of combined scores in NSCLC $(n=165)$

\begin{tabular}{|c|c|c|c|c|c|c|c|c|}
\hline \multirow{2}{*}{ Type } & \multicolumn{4}{|c|}{$\begin{array}{c}\text { Combined score } \\
n(\%)\end{array}$} & \multicolumn{3}{|c|}{$\begin{array}{c}\text { Groupings } \\
n(\%)\end{array}$} & \multirow{2}{*}{$p$-value } \\
\hline & 0 & 1 & 2 & 3 & & $0-1$ & $2-3$ & \\
\hline $\operatorname{SCC}(n=97)$ & $0(0)$ & $67(69)$ & $25(26)$ & $5(5)$ & $<0.001$ & $67(69)$ & $30(31)$ & $<0.001$ \\
\hline $\operatorname{ADC}(n=54)$ & $1(2)$ & $0(0)$ & $44(82)$ & $9(16)$ & & $1(2)$ & $53(98)$ & \\
\hline $\operatorname{LCC}(n=10)$ & $3(30)$ & $2(20)$ & $4(40)$ & $1(10)$ & & $5(50)$ & $5(50)$ & \\
\hline $\operatorname{ADSCC}(n=4)$ & $0(0)$ & $0(0)$ & $4(100)$ & $0(0)$ & & $0(0)$ & $4(100)$ & \\
\hline
\end{tabular}

${ }^{a}$ Degree of immunohistochemical expression using the combination of extent and intensity of the staining; combined score $0(0)$, combined score 1 (1-2), combined score 2 (3-4), combined score 3 (5-6).

MUC, mucin; NSCLC, non-small cell lung carcinoma; SCC, squamous cell carcinoma; ADC, adenocarcinoma; LCC, large cell carcinoma; ADSCC, adenosquamous carcinoma.

Table 2. Comparison of MUC4 expression between each combined score and groupings of combined scores in NSCLC $(n=165)$

\begin{tabular}{|c|c|c|c|c|c|c|c|c|}
\hline \multirow[t]{2}{*}{ Type } & \multicolumn{4}{|c|}{$\begin{array}{c}\text { Combined score } \\
n(\%)\end{array}$} & \multicolumn{3}{|c|}{$\begin{array}{l}\text { Groupings } \\
n(\%)\end{array}$} & \multirow{2}{*}{$\mathrm{p}$-value } \\
\hline & 0 & 1 & 2 & 3 & & $0-1$ & $2-3$ & \\
\hline $\operatorname{SCC}(n=97)$ & $36(37)$ & $54(56)$ & $6(6)$ & $1(1)$ & $<0.05$ & $90(93)$ & $7(7)$ & $<0.01$ \\
\hline$A D C(n=54)$ & $15(28)$ & $23(43)$ & $12(22)$ & $4(7)$ & & $38(71)$ & $16(29)$ & \\
\hline $\operatorname{LCC}(n=10)$ & $5(50)$ & $4(40)$ & $0(0)$ & $1(10)$ & & $9(90)$ & $1(10)$ & \\
\hline $\operatorname{ADSCC}(n=4)$ & $1(25)$ & $3(75)$ & $0(0)$ & $0(0)$ & & $4(100)$ & $0(0)$ & \\
\hline
\end{tabular}

${ }^{a}$ Degree of immunohistochemical expression using the combination of extent and intensity of the staining; combined score 0 (0), combined score 1 (1-2), combined score 2 (3-4), combined score 3 (5-6).

MUC, mucin; NSCLC, non-small cell lung carcinoma; SCC, squamous cell carcinoma; ADC, adenocarcinoma; LCC, large cell carcinoma; ADSCC, adenosquamous carcinoma. 


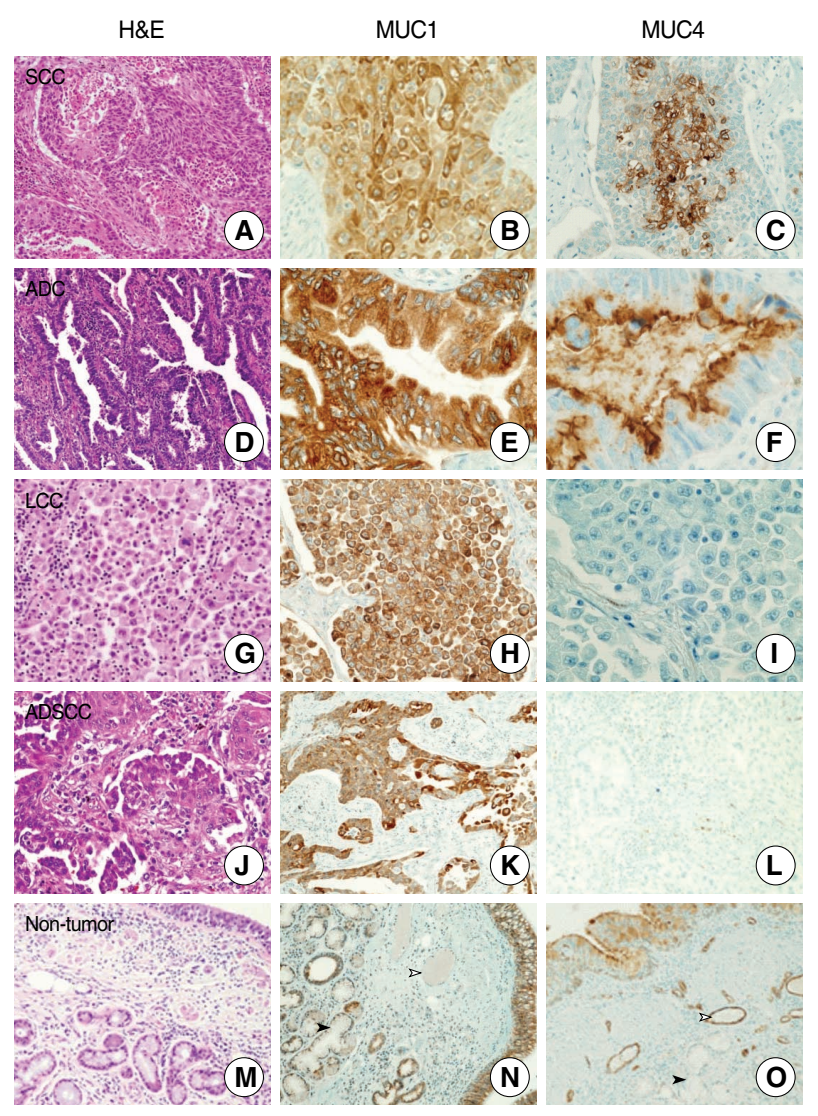

Fig. 2. Mucin (MUC)1 and MUC4 proteins show various expression patterns in different non-small cell lung carcinoma histologic subtype. (A-C) Squamous cell carcinoma (SCC): MUC1 shows diffuse expression in the tumor cells (B), but MUC4 shows well differentiated tumor cells, especially the central portion of the tumor nest (C). (D-F) Adenocarcinoma (ADC): MUC1 shows diffuse cytoplasmic expression (E), but MUC4 shows expression in the apical and luminal portion of the tumor gland (F). (G-I) Large cell carcinoma (LCC): MUC1 shows diffuse expression in the tumor cells $(\mathrm{H})$, but MUC4 shows no expression (I). (J-L) Adenosquamous carcinoma (ADSCC): MUC1 shows positive expression in the tumor cells of both SCC and ADC (K), but MUC4 shows no expression in the tumor cells (L). (M-O) Non-tumor: MUC1 shows positive expression in the bronchial epithelial cells and submucosal glands, but no expression in the vascular endothelial cells (N). In contrast MUC4 shows positive expression in the bronchial epithelial cells and vascular endothelial cells, but no expression in the submucosal glands $(\mathrm{O})$. White arrow head, vascular structure; Black arrow head, submucosal glands; H\&E, hematoxylin and eosin.

\section{Statistical analysis of MUC1 and MUC4 expression in NSCLC}

There were no statistically significant associations between MUC1 or MUC4 expression levels and patient gender, age at the time of diagnosis and pathological stage. However, MUC1 and MUC4 expression levels were significantly correlated with the histologic subtype of NSCLC. High expression of MUC1 was characteristic in adenocarcinoma, with combined scores of 2 and $3(98 \%, \mathrm{p}<0.001)$ (Table 1). In contrast, low expression levels of MUC1 and MUC4 were characteristic of squamous cell carcinoma, with combined scores of 0 and 1 (69\% and 93\%, respectively) (Tables 1,2). Statistical analyses of MUC1 and MUC4 expression levels were not available for large cell carcinoma and adenosquamous carcinoma due to insufficient numbers.

\section{Prognostic significance of MUC1 and MUC4 expression in NSCLC}

We performed survival analysis in patients with squamous cell carcinoma, adenocarcinoma, and other NSCLCs. There were no prognostic significances according to the combined scores of MUC1 and MUC4. However, there were some prognostic significance according to the extent scores of MUC1 and MUC4. Survival analysis showed that diffuse MUC1 expression (extent score 3) was significantly associated with shortened survival in NSCLC patients, excluding cases of squamous cell carcinoma $(\mathrm{p}=0.005)$ (Fig. 3A). There was no significant correlation between diffuse MUC1 expression and shortened patient survival in squamous cell carcinoma (Fig. 3B). There was a trend of longer patient survival in NSCLC patients with diffuse MUC4 expression (extent score 2 and 3 ), excluding squamous cell carcinoma $(\mathrm{p}=0.128)$ (Fig. 4A). The survival analysis for diffuse MUC4 expression (extent score 2 and 3 ) showed no significant association in squamous cell carcinoma (Fig. 4B), which was similar to the result for MUC1 expression. No significant correlation was detected between high MUC1 and MUC4 expression levels and patient survival in NSCLC, adenocarcinoma, and large cell carcinoma ( $p>0.05)$. After controlling for disease stage, there was no significant association between high MUC1 and MUC4 expressions and patient survival in patients with stage I and II tumors.

\section{DISCUSSION}

This study demonstrated high expression levels of MUC1 and MUC4 in NSCLC, with more frequent detection of MUC1 than MUC4. Our results support a previous study by Seregni et al. ${ }^{17}$ demonstrated by northern blot analysis that MUC1 was the highest expressed $M U C$ gene in most lung carcinomas, followed by MUC4.

MUCs are high-molecular weight glycoconjugates (154 to 

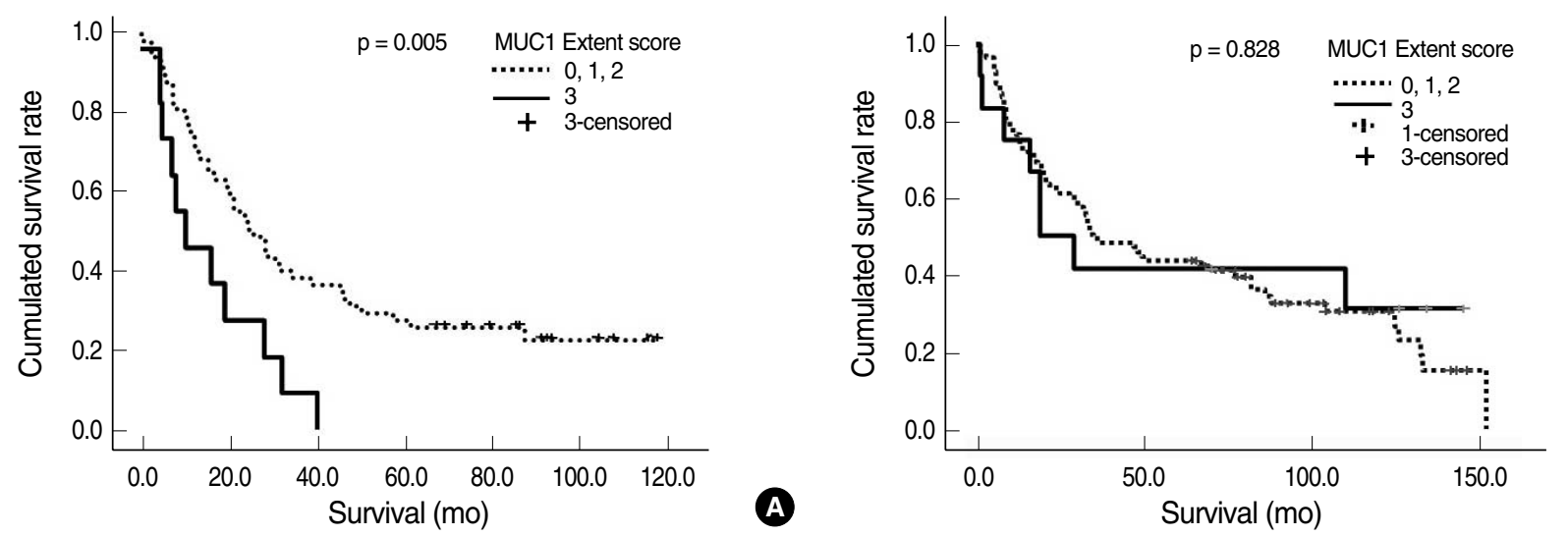

B

Fig. 3. Kaplan-Meier survival curves for mucin (MUC)1 expression according to non-small cell lung carcinoma (NSCLC) histologic subtype. (A) In NSCLC, excluding cases of squamous cell carcinoma, there is a significant correlation between diffuse MUC1 expression (extent score 3 ) and shortened patient survival ( $p=0.005)$. (B) In squamous cell carcinoma, there is no significant association between diffuse MUC1 expression (extent score 3 ) and patient survival $(p=0.828)$.
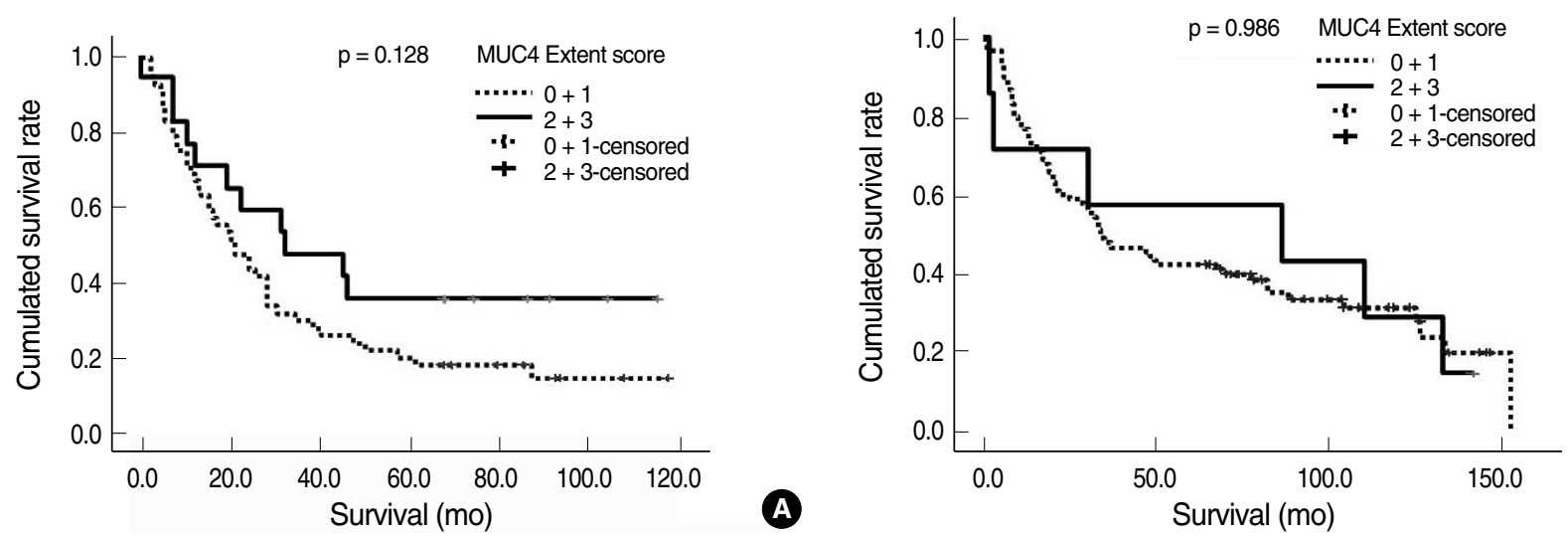

Fig. 4. Kaplan-Meier survival curves for mucin (MUC)4 expression according to non-small cell lung carcinoma (NSCLC) histologic subtype. (A) In NSCLC, excluding squamous cell carcinoma, Kaplan-Meier analysis reveals a trend toward improved survival in association with increased MUC4 expression (extent score 2 and 3) $(p=0.128$ ). (B) In squamous cell carcinoma, there is no significant association between increased MUC4 expression (extent score 2 and 3 ) and patient survival $(p=0.986)$.

over 7,000 kDa) with hundreds of oligosaccharide chains in Oglycosidic linkages to a protein backbone. MUC1 is a transmembrane-bound MUC with a transmembrane segment, followed by a 69-amino acid cytoplasmic tail at the amino terminal end. Maeshima et al..$^{18}$ reported high frequency of MUC1 expression in adenocarcinoma compared to other types of lung cancer. This study showed that high MUC1 expression was characteristic of adenocarcinoma, whereas low expression of MUC1 and MUC4 was characteristic of squamous cell carcinoma. Maeshima et al. ${ }^{18}$ reported that high MUC1 expression was associated with poor survival in patients with NSCLC. Tsuda $e t$ al. ${ }^{19}$ reported that high MUC1 gene expression was correlated with poor survival in 38 patients with NSCLC. Several studies suggested that MUC1 expression was an important component of biochemical events that enabled metastasis in cancer cells. ${ }^{20-22}$
Wesseling et al. ${ }^{23}$ showed that MUC1 inhibited E-cadherin mediated the cell-cell adhesion system, and inhibition is dependent on the length of MUC1 ectodomain. High MUC1 levels also reduced integrin mediated cell adhesion to the extracellular matrix. ${ }^{24} \mathrm{MUC1}$ expressing cells are less susceptible to $\mathrm{T}$ cell mediated lysis, which may contribute to the ability of cancer cells to escape from immune surveillance. ${ }^{25}$

MUC4 is a heterodimeric glycoprotein complex consists of a MUC subunit, MUC4 alpha (ASGP-1 in the rat), tightly bound to a transmembrane subunit, MUC4 beta (ASGP-2 in the rat). ${ }^{26}$ The relationship between MUC4 expression and tumor behavior appears to be tissue-dependent. ${ }^{12,13,26-28}$ MUC4 over-expression was associated with aggressiveness and metastasis in breast cancer, extrahepatic bile duct carcinoma, and cholangiocarcinoma. ${ }^{12,13,27}$ Conversely, improved patient survival was associated 
with high MUC4 expression in ovarian cancer, mucoepidermoid carcinoma of the salivary glands, and squamous cell carcinoma of the upper aerodigestive tract. ${ }^{26,28}$ These divergent clinical behaviors may result from tissue and tumor-specific differences in the relative importance of differentiation versus proliferative signals mediated by MUC4. Animal studies elucidating the functional relationship between MUC4 and ErbB2 suggested that these two membrane molecules associate as ligand and receptor under various conditions, mediating different downstream signaling pathways. ${ }^{29}$ Two pathways of MUC4-mediated ErbB2 tyrosine phosphorylation have been demonstrated. Phosphorylation of the MUC4-ErbB2 complex can occur in the absence of heterodimerization with other receptors of the ErbB family, and in the absence of other known soluble ligands. ${ }^{30}$ The heterodimers formed by MUC4-activated ErbB2 and neuregulin-activated ErbB3 are characterized by potentiated phosphorylation of each of the $2 \mathrm{ErbB}$ receptors, compared with MUC4-ErbB2 or ErbB2-ErbB3-neuregulin complex.

This study investigated the correlation between the expression of both membrane-bound MUCs (MUC1 and MUC4) and NSCLC prognosis. We found that diffuse MUC1 expression significantly correlated with shortened survival in NSCLC, excluding squamous cell carcinoma. However, diffuse MUC4 expression was associated with longer survival in NSCLC, excluding squamous cell carcinoma. In adenocarcinoma, our results on the relationship between diffuse expression of MUC1, and MUC4 and survival were not consistent with previous reports. It remains unclear whether the expression of MUC1 and MUC4 proteins in lung carcinoma cells correlates with mRNA expression. We speculated that increased expression of MUC1 and MUC4 proteins was either due to increased mRNA levels, or to alterations of MUC1 and MUC4 glycosylation. Future studies in our laboratory will focus on the correlation between mRNA expression and the protein expression of MUC1 and MUC4 in NSCLC.

In conclusion, the expression levels of MUC1 and MUC4 were significantly correlated with the NSCLC histologic subtype. Diffuse MUC1 expression was significantly correlated with shortened survival in NSCLC, excluding squamous cell carcinoma. In addition, diffuse MUC4 expression showed longer survival trend in NSCLC, excluding squamous cell carcinom.

\section{REFERENCES}

1. Kelsey CR, Boyd JA, Hubbs JL, et al. Local/regional recurrence following surgery for early-stage lung cancer: a 10-year experience with 975 patients. J Clin Oncol 2008; 26(15 Suppl): 7542.

2. Liu XQ, Ren HX, Wu ZP. Detecting MUC-1 mRNA for diagnosing peripheral blood micro-metastasis in non-small cell lung cancer patients. Ai Zheng 2008; 27: 1267-70.

3. Komatsu M, Tatum L, Altman NH, Carothers Carraway CA, Carraway KL. Potentiation of metastasis by cell surface sialomucin complex (rat MUC4), a multifunctional anti-adhesive glycoprotein. Int J Cancer 2000; 87: 480-6.

4. Moniaux N, Escande F, Porchet N, Aubert JP, Batra SK. Structural organization and classification of the human mucin genes. Front Biosci 2001; 6: D1192-206.

5. Senapati S, Sharma P, Bafna S, Roy HK, Batra SK. The MUC gene family: their role in the diagnosis and prognosis of gastric cancer. Histol Histopathol 2008; 23: 1541-52.

6. Nakamori S, Ota DM, Cleary KR, Shirotani K, Irimura T. MUC1 mucin expression as a marker of progression and metastasis of human colorectal carcinoma. Gastroenterology 1994; 106: 353-61.

7. Ho SB, Niehans GA, Lyftogt C, et al. Heterogeneity of mucin gene expression in normal and neoplastic tissues. Cancer Res 1993; 53: 641-51.

8. Okabe S, Ishikawa T, Tanami S, et al. Clinicopathological study of mucous pooling referred to as mucinous component (MUC) in colorectal submucosal invasive carcinomas. J Med Dent Sci 2001; 48: 69-78.

9. Monges GM, Mathoulin-Portier MP, Acres RB, et al. Differential MUC 1 expression in normal and neoplastic human pancreatic tissue: an immunohistochemical study of 60 samples. Am J Clin Pathol 1999; 112: 635-40.

10. Khodarev NN, Pitroda SP, Beckett MA, et al. MUC1-induced transcriptional programs associated with tumorigenesis predict outcome in breast and lung cancer. Cancer Res 2009; 69: 2833-7.

11. Andrianifahanana M, Moniaux N, Schmied BM, et al. Mucin (MUC) gene expression in human pancreatic adenocarcinoma and chronic pancreatitis: a potential role of MUC4 as a tumor marker of diagnostic significance. Clin Cancer Res 2001; 7: 4033-40.

12. Carraway KL, Price-Schiavi SA, Komatsu M, Jepson S, Perez A, Carraway CA. Muc4/sialomucin complex in the mammary gland and breast cancer. J Mammary Gland Biol Neoplasia 2001; 6: 323-37.

13. Shibahara H, Tamada S, Higashi M, et al. MUC4 is a novel prognostic factor of intrahepatic cholangiocarcinoma-mass forming type. Hepatology 2004; 39: 220-9.

14. Hanaoka J, Kontani K, Sawai S, et al. Analysis of MUC4 mucin expression in lung carcinoma cells and its immunogenicity. Cancer 2001; 92: 2148-57.

15. Travis WD, Brambilla E, Muller-Hermelink HK, Harris CC. World Health Organization classification of tumours: pathology and genet- 
ics of tumours of the lungs, pleura, thymus and heart. Lyon: IARC Press, 2004.

16. Goldstraw P, Crowley J, Chansky K, et al. The IASLC Lung Cancer Staging Project: proposals for the revision of the TNM stage groupings in the forthcoming (seventh) edition of the TNM classification of malignant tumours. J Thorac Oncol 2007; 2: 706-14.

17. Seregni E, Botti C, Lombardo C, et al. Pattern of mucin gene expression in normal and neoplastic lung tissues. Anticancer Res 1996; 16: 2209-13.

18. Maeshima A, Miyagi A, Hirai T, Nakajima T. Mucin-producing adenocarcinoma of the lung, with special reference to goblet cell type adenocarcinoma: immunohistochemical observation and Kiras gene mutation. Pathol Int 1997; 47: 454-60.

19. Tsuda T, Mitsudomi T, Ohgami A, et al. Mucin gene expression is associated with the poor prognosis in human non-small cell lung cancer. Proc Am Assoc Cancer Res 1995; 36: 214.

20. Zhao Q, Guo X, Nash GB, et al. Circulating galectin-3 promotes metastasis by modifying MUC1 localization on cancer cell surface. Cancer Res 2009; 69: 6799-806.

21. Nogi H, Takeyama H, Uchida K, Agata T, Horiguchi-Yamada J, Yamada H. Detection of MUC1 and keratin 19 mRNAs in the bone marrow by quantitative RT-PCR predicts the risk of distant metastasis in breast cancer patients. Breast Cancer 2003; 10: 74-81.

22. Nitta T, Sugihara K, Tsuyama S, Murata F. Immunohistochemical study of MUC1 mucin in premalignant oral lesions and oral squamous cell carcinoma: association with disease progression, mode of invasion, and lymph node metastasis. Cancer 2000; 88: 245-54.

23. Wesseling J, van der Valk SW, Hilkens J. A mechanism for inhibi- tion of E-cadherin-mediated cell-cell adhesion by the membraneassociated mucin episialin/MUC1. Mol Biol Cell 1996; 7: 565-77.

24. Wesseling J, van der Valk SW, Vos HL, Sonnenberg A, Hilkens J. Episialin (MUC1) overexpression inhibits integrin-mediated cell adhesion to extracellular matrix components. J Cell Biol 1995; 129: 255-65.

25. van de Wiel-van Kemenade E, Ligtenberg MJ, de Boer AJ, et al. Episialin (MUC1) inhibits cytotoxic lymphocyte-target cell interaction. J Immunol 1993; 151: 767-76.

26. Weed DT, Gomez-Fernandez C, Yasin M, et al. MUC4 and ErbB2 expression in squamous cell carcinoma of the upper aerodigestive tract: correlation with clinical outcomes. Laryngoscope 2004; 114: 132.

27. Takao S, Uchikura K, Yonezawa S, Shinchi H, Aikou T. Mucin core protein expression in extrahepatic bile duct carcinoma is associated with metastases to the liver and poor prognosis. Cancer 1999; 86: 1966-75.

28. Weed DT, Gomez-Fernandez C, Pacheco J, et al. MUC4 and ERBB2 expression in major and minor salivary gland mucoepidermoid carcinoma. Head Neck 2004; 26: 353-64.

29. Theodoropoulos G, Carraway CA, Carraway KL. MUC4 involvement in ErbB2/ErbB3 phosphorylation and signaling in response to airway cell mechanical injury. J Cell Biochem 2009; 107: 112-22.

30. Ramsauer VP, Pino V, Farooq A, Carothers Carraway CA, Salas PJ, Carraway KL. Muc4-ErbB2 complex formation and signaling in polarized CACO-2 epithelial cells indicate that Muc4 acts as an unorthodox ligand for ErbB2. Mol Biol Cell 2006; 17: 2931-41. 